\title{
Estudo de diversos conceitos de eficiência da locomoção humana no meio aquático
}

\author{
Tiago M. Barbosa ${ }^{1}$ \\ João P. Vilas-Boas ${ }^{2}$
}

https://doi.org/10.5628/rpcd.05.03.337

\author{
${ }^{1}$ Instituto Politécnico de Bragança \\ Escola Superior de Educação \\ 2 Universidade do Porto \\ Faculdade de Desporto \\ Portugal
}

\section{RESUMO}

Uma das áreas de interesse na linha fronteira entre a Biomecânica e a Fisiologia é o estudo da eficiência. Considerase que a eficiência expressa uma relação objectiva e quantitativa entre o trabalho mecânico realizado e o gasto energético que se lhe encontra associado. O estudo da eficiência em actividades realizadas no meio aquático torna-se ainda mais comple xo devido à dificuldade de quantificar objectivamente as trocas energéticas entre o nadador e o meio envolvente. No entanto, a Natação Pura Desportiva é uma das modalidades mais profícuas no estudo das suas implicações bioenergéticas e das repercussões em termos de eficiência dos diferentes processos de locomoção. Assim, foi objectivo do presente trabalho efectuar uma revisão do actual "estado da arte" sobre o estudo da locomoção humana no meio aquático, com especial referência às técnicas de nado.

Palavras-chave: eficiência, locomoção, meio aquático, natação.

\begin{abstract}
Study of several efficiency concepts about human locomotion in aquatic environment

One of the major interests in the frontier between Biomechanics and Physiology is the study of efficiency. The efficiency expresses the relationship between the mechanical work and the related energy expenditure. The study of efficiency, in the water, becomes more complex due to difficulties in quantifying, with accuracy, the energetic exchanges between the swimmer and the environment. However, swimming is one of the most studied sports, in order to understand its bioenergetical implications and the efficiency's repercussions of different locomotion processes. "State of the art" about human locomotion in water, with special reference to swimming techniques is thus presented.
\end{abstract}

Key Words: efficiency, locomotion, aquatic environment, swimming. 


\section{CONCEITO DE EFICIÊNCIA}

Uma das áreas de interesse na intercepção mecânico-fisiológica é o estudo da eficiência. Em diversos conte xtos, um dos objectivos prioritários da Biomecânica será o de, num primeiro momento, caracterizar um determinado padrão motor e, num segundo momento, inter vir no sentido de incrementar a eficiência da sua execução. Para tal, considerase que a eficiência expressa uma relação objectiva e quantitativa entre o trabalho mecânico realizado e o gasto energético que se lhe encontra associado (10). $\mathrm{O}$ conceito de eficiência tem origem nos sistemas mecânicos, especialmente afectos à Engenharia. No entanto, no domínio da actividade física, a eficiência de uma determinada tarefa, expressa a razão entre o somatório de todos os trabalhos internos e externos realizados e o custo metabólico que lhe está associado (1).
Winter (48) descreveu o fluxo da energia desde a sua entrada num sistema vivo (input) até à sua saída (output) sob a forma de trabalho mecânico externo. A figura 1 apresenta esse fluxo da energia ao longo do percurso. A produção de energia metabólica decorre do consumo de oxigénio e da produção de dióxido de carbono. Parte dessa energia é perdida com vista à manutenção do calor corporal, outra parte devido à própria contracção muscular . A energia restante será utilizada para desenvolver tensão muscular, inclusivamente, para a realização de trabalho isométrico postural. Neste percurso, uma percentagem da energia é perdida devido a outros factores, como sejam a co-contracção ou a absorção de energia pelos músculos. Será a energia que não foi utilizada até ao momento a disponibilizada para promover um incremento da energia segmentar, resultando na produção de trabalho mecânico externo.

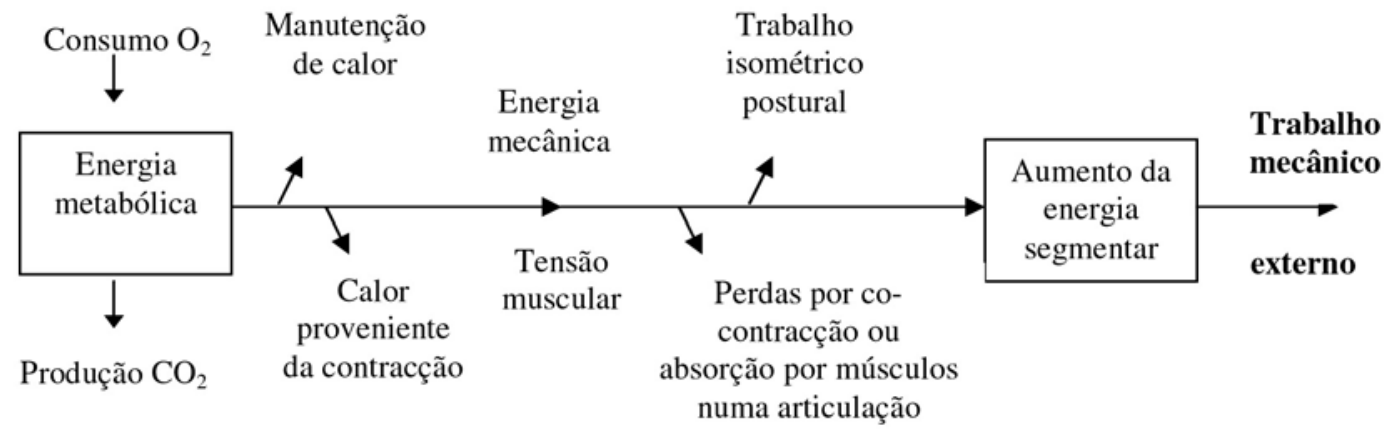

Figura 1. Fluxo de energia desde a entrada no sistema - potência metabólica - até à sua saída - trabalho mecânico externo (adaptado de Winter, 48).

Do exposto emerge desde logo que, num âmbito epistemológico, o estudo da eficiência encontra-se numa linha fronteira entre a Biomecânica e a Fisiologia $(7,32)$. O desenvolvimento de investigação nesta área carece da análise de variáveis bioenergéticas como, por exemplo, o consumo de oxigénio ou as concentrações séricas de lactato; mas também da análise das repercussões de variáveis biomecânicas - como sejam, a título ilustrativo, a velocidade de deslocamento, a mecânica do ciclo gestual ou a flutuação intracíclica da velocidade horizontal de deslocamento do centro de massa - nesses parâmetros bioenergéticos.
Caso paradigmático será o estudo do custo energético de locomoção (C). Este parâmetro é calculado com base numa variável bioenergética, a potência metabólica do sistema (Pmet) e numa variável biomecânica, a velocidade de deslocamento do corpo (v):

$\mathrm{C}=\frac{\text { Pmet }}{\mathrm{v}}$

Desta feita, [C] representa a energia despendida para percorrer uma determinada distância (16-18, 32, 4951). Portanto, assume-se que um aumento do [C] implicará uma diminuição da eficiência de locomoção do corpo na tarefa em análise. 
Ou seja, verifica-se claramente o estabelecimento de pontes entre a Mecânica Clássica com a

Termodinâmica e a Bioenergética (45). Estas pontes pressupõem o entendimento do organismo biológico como um sistema termodinâmico e o desempenho desportivo como uma emergência de processos optimizados de aporte energético, repercussão das características biomecânicas desse sistema (45).

$\mathrm{Na}$ maioria das actividades desportivas orientadas para o rendimento o objectivo final da sua prática é a obtenção do melhor resultado desportivo possível em conte xto competitivo. No caso da Natação Pura Desportiva (NPD), o carácter claramente temporal e espacial do seu rendimento, permite uma quantificação energética precisa da mesma (4).

A NPD é uma das modalidades mais profícuas no estudo das suas implicações bioenergéticas e das repercussões em termos de eficiência de diferentes modelos de locomoção. Todavia, o estudo da eficiência em actividades realizadas no meio aquático, como é o caso da NPD, torna ainda mais comple xo este tipo de análise, devido à dificuldade em quantificar objectivamente as trocas energéticas entre o nadador e o meio envolvente.

A evolução do conceito de eficiência de locomoção aquática do Homem tem sido bastante rápido. Talvez devido ao fascínio que as actividades aquáticas induzem (32). Possivelmente devido às implicações práticas que tem no domínio do controlo e da avaliação do treino na NPD e no desempenho dos nadadores em situação competitiva (18).

Num quadro histórico, Hudson (23) distingue duas fases na evolução da Biomecânica. A Biomecânica pré-moderna (entre 1940 e 1965), associada à Cinesiologia e com forte influência da Anatomia, da Fisiologia e da Mecânica. O estudo da cinemática segmentar e dos padrões de movimento assumiam especial relevância neste período. A segunda fase é a da Biomecânica moderna (desde 1965 até, pelo menos, aos nossos dias) alicerçada na Mecânica e no desenvolvimento tecnológico que permite a utilização de instrumentos cada vez mais sofisticados. Esta fase caracteriza-se pelo complemento dos estudos cinemáticos com a dinâmica e pelo estabelecimento de pontes com a Termodinâmica e a Bioenergética. Assim, há que assumir que, numa perspectiva histórica, este fascínio pelo estudo da eficiência da loco- moção é um tanto recente. Pelo que não será arriscar muito afirmar que, só agora, se começa a dar os primeiros passos na consolidação dos conhecimentos inerentes a este campo de investigação.

Assim foi objectivo do presente trabalho efectuar uma revisão do actual "estado da arte" sobre o estudo da locomoção humana no meio aquático, com especial referência às técnicas de nado. Isto é, efectuar uma revisão conceptual sobre o actual entendimento relativo à eficiência de locomoção humana no meio aquático, norteando esta apresentação na relação que se estabelece entre as concepções biomecânicas e bioenergéticas.

\section{FACTORES DETERMINANTES DO DESEMPENHO COMPETITIVO EM NATAÇÃO}

Um dos propósitos dos nadadores, em situação competitiva, é o de atingirem elevadas velocidades de deslocamento. Um dos pioneiros no estudo dos princípios mecânicos subjacentes ao nado foi Counsilman (13). Segundo o autor, a velocidade de nado, em dado momento, é o resultado da interacção entre a força de arrasto e a propulsão. Um nadador poderá incrementar a sua velocidade de deslocamento a partir de três estratégias (13): (i) diminuindo a força de arrasto a que se encontra submetido; (ii) aumentando a produção de força propulsiva ou; (iii) realizando uma combinação das duas situações atrás descritas. Mais tarde, Craig (12), acrescenta outros factores determinantes para o incremento da velocidade de nado:

$\mathrm{v}=\frac{\text { Pmet.e }}{\mathrm{D}}$

Onde [v] é a velocidade de deslocamento do nadador, [Pmet] a potência metabólica, [e] a eficiência e [D] a força de arrasto hidrodinâmico, considerando em todas as variáveis a análise do centro de massa do sujeito. Quer isto dizer que a velocidade de nado é directamente proporcional à taxa de produção de energia metabólica num dado inter valo de tempo e à eficiência, mas inversamente proporcional à força de arrasto hidrodinâmico.

Toussaint (41) actualizou e explorou um pouco mais este conceito. A velocidade de nado depende de cinco factores fundamentais: a força de arrasto 
hidrodinâmico, a taxa de entrada de energia no sistema (potência metabólica), a eficiência mecânica, a eficiência propulsiva e a taxa de energia produzida pelo sujeito (potência mecânica externa). A figura 2 apresenta os factores determinantes da velocidade de nado, de acordo com Toussaint (41).

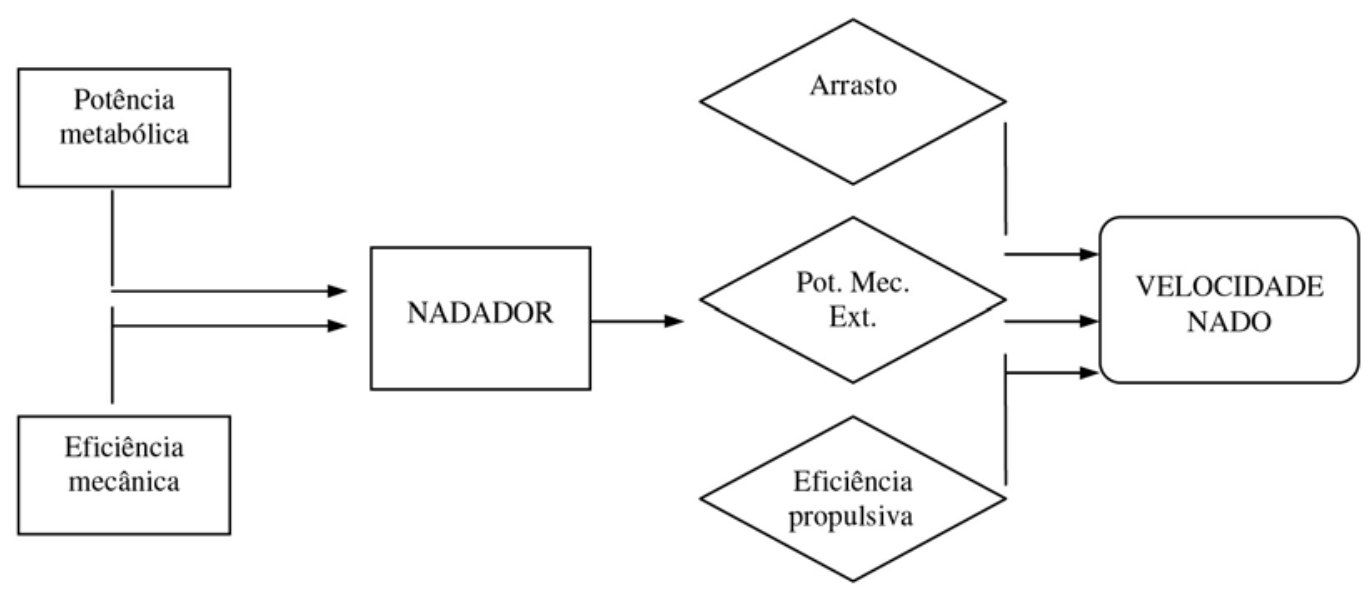

Figura 2. Determinantes da velocidade de nado em Natação Pura Desportiva (adaptado de Toussaint, 41).

De seguida serão dissecados os conceitos e os pressupostos associados a cada um dos factores determinantes da velocidade de nado em NPD, com base no modelo de Toussaint (41).

\subsection{A potência metabólica}

Qualquer gesto técnico realiza-se fundamentalmente devido à capacidade do organismo extrair energia dos alimentos (energia química) e de os transformar em energia mecânica (6). É a partir da potência metabólica que ocorre a entrada de energia no sistema.

Os músculos tem de produzir potência para deslocar o corpo. A produção total de [Pmet] determina-se a partir do somatório da produção energética decorrente da degradação do ATP-CP, da glicólise anaeróbia e do sistema aeróbio $(31,42)$ em que:

Paer $=$ Paer,max $\left(1-\mathrm{e}^{-\lambda t}\right)$

e

Pan $=$ Pan, $\max \cdot \mathrm{e}^{-\lambda \mathrm{t}}$

Onde [Paer] é a potência aeróbia, [Pan] a potência anaeróbia, [Paer,max] é a potência aeróbia máxima, [Pan,max] a potência anaeróbia máxima, [t] o tempo, $[\lambda]$ uma constante que expressa o aumento ou a diminuição da potência e [e] a eficiência.
Para a maioria das provas que constituem o quadro competitivo da NPD, o contributo do sistema ATP$\mathrm{CP}$ para a [Pmet] é negligenciável (33). Dado que a duração da maioria das provas oficiais de NPD privilegia a produção de energia a partir dos sistemas aeróbio e anaeróbio, considera-se o contributo percentual do sistema ATP-CP como sendo pouco significativo para o valor total da [Pmet].

Consequentemente, a [Pmet] decorre do somatório da potência aeróbia com a potência anaeróbia:

Pmet $=$ Paer + Pan

Desta forma, a quantidade total de energia aeróbia ou anaeróbia despendida num dado inter valo de tempo pode ser calculada integrando a respectiva potência (38):

Eaer $=\int_{0}^{1}$ Paer, $\max \left(1-\mathrm{e}^{-\mathrm{t} t}\right) \mathrm{dt}=$ Paer, $\max \cdot \mathrm{t}+\frac{\text { Paer, max }}{\ddot{\mathrm{e}}} \cdot\left(\mathrm{e}^{-\mathrm{t} t}-1\right)$

e

Ean $=\int_{0}^{t}$ Pan, $\max \cdot e^{-\bar{e} t} d t=\frac{\text { Pan, max }}{\ddot{e}} \cdot\left(1-e^{-\bar{e} t}\right)$ 
Onde [Eaer] é a quantidade total de energia aeróbia produzida e [Ean] a quantidade de energia anaeróbia produzida.

\subsection{A eficiência mecânica}

Para a realização de trabalho mecânico, nem toda a produção da [Pmet] será utilizada (6). Em virtude deste ser um processo endotrópico, parte da [Pmet] é utilizada para a produção de energia térmica (6, 10, 14). Assim, só uma fracção da [Pmet] é que será dispensada para a produção de potência mecânica externa (Pext).

Dado que só uma porção da [Pmet] é utilizada em [Pext], é possível determinar a taxa de [Pmet] utilizada para esse mesmo fim. Consequentemente, a eficiência mecânica (em) expressa a razão entre a [Pext] e a [Pmet] $(36,39,41)$ :

$\mathrm{em}=\frac{\text { Pext }}{\text { Pmet }} .100$

Toussaint (36, 38, 41) e Toussaint et al. (39), demostraram que na técnica de Crol, a [Pext] e a [Pmet] se correlacionavam positivamente e com elevado significado estatístico (entre os $\mathrm{r}=0.89$ e os $\mathrm{r}=0.94)$. Mais, os valores $\mathrm{da}[\mathrm{em}]$, nessa mesma técnica de nado, oscilavam entre os $8.5 \%$ e os $9.7 \%$. Ou seja, de toda a [Pmet], apenas $10 \%$ são utilizados em [Pext]. Os restantes $90 \%$ são despendidos fundamentalmente através de processos associados à termoregulação.

Di Prampero et al. (16) avaliaram o consumo líquido de oxigénio, a diferentes velocidades, à medida que os nadadores eram puxados para a frente (arrasto negativo) ou para trás (arrasto positivo) através de um sistema de cargas e roldanas por onde passava um cabo ligado ao nadador. Através da recta de regressão entre o consumo líquido de oxigénio e o valor das diferentes cargas, foi possível extrapolar o valor do arrasto para um consumo nulo. Esse valor da carga define o arrasto a que o nadador está sujeito a uma dada velocidade. Assumindo que a velocidade é constante, a [em] pode ser calculada a partir do declive da recta de regressão previamente definida e pela velocidade de deslocamento. Verificou-se que a [em] variou entre os $1.0 \%$ e os $7.5 \%$ (16). Estes valores parecem ser dramaticamente reduzi- dos, quando comparados com outras actividades como a corrida (34 a 45\%), a marcha (20 a 35\%) ou o ciclismo (24 a 30\%), de acordo com Williams (47). Isto é, na NPD é reconduzida, para as questões associadas à regulação térmica, uma percentagem mais elevada da [Pmet] do que nas actividades realizadas no meio terrestre.

Posteriormente, Kemper et al. (26), ao estudarem 50 estudantes universitários de Educação Física, verificaram que a [em] variou entre os $1.99 \%$ e os $8.33 \%$. Por outras palavras, parece ser consensual o facto da [em] em NPD ser bastante reduzida.

Uma outra característica da [em] é que não é um parâmetro fortemente discriminativo do nível de desempenho dos sujeitos. Não se verificaram diferenças significativas na [em] entre nadadores de competição e de recreio (11). Kemper et al. (26), ao compararem dois grupos de nadadores com níveis de desempenho diferentes, não verificaram diferenças substanciais entre eles. No mesmo sentido, Toussaint (38) também não obser vou diferenças estatisticamente significativas da [em] entre nadadores de elite e triatletas.

Relativamente a uma possível variabilidade intersexual da $[\mathrm{em}]$, mais uma vez parece não existirem diferenças significativas, caso ambos os grupos apresentem valores idênticos de [Pext] (39). Pendergast et al. (30) e di Prampero (17) atribuíram um menor custo energético obser vado (em valores próximos dos 30\%) em nadadoras do que em nadadores a diferenças na $[\mathrm{em}]$. Todavia, possivelmente a causa efectiva terá sido o arrasto total que foi inferior em $29 \%$ nas nadadoras do que nos nadadores, quando nadavam a uma velocidade de $1.0 \mathrm{~m} . \mathrm{s}^{-1}$. Para tal, há que considerar que o valor do arrasto não foi relativizado à massa corporal, estando -se portanto a falar de valores absolutos.

Estudando a variação da [em] com base nas diferentes técnicas de nado, o valor mais elevado surgiu na técnica de crol, variando entre os $6.7 \%(16,21)$ e os $15 \%$ (31). Na técnica de bruços o valor foi ligeiramente inferior, variando entre os $4 \%$ e os $6 \%$ (21, 22). A razão para tão reduzida $[\mathrm{em}]$ da técnica de bruços será a forte dependência da propulsão gerada a partir dos membros inferiores. A técnica de mariposa, de entre as quatro técnicas de nado formal, foi aquela com a menor $[\mathrm{em}](16,21)$. Nas técnicas 
simultâneas as condições de execução impõem um ritmo e uma sequência de acções propulsivas descontínuas, levando a acelerações e desacelerações acentuadas, as quais são menos favoráveis em termos de aproveitamento energético. Depois destes estudos não foram realizados outros, pelo que os dados devem ser analisados cautelosamente, dada a evolução que as técnicas de nado entretanto apresentaram.

Procurando aferir a variação da [em] de acordo com a utilização de equipamentos, como barbatanas, mais uma vez não se verificam diferenças significativas. Zamparo et al. (51) constataram que ao nadar, entre os $0.6 \mathrm{~m} . \mathrm{s}^{-1}$ e os $1.0 \mathrm{~m} . \mathrm{s}^{-1}$, com barbatanas pequenas e flexíveis a [em] foi de $13 \pm 2 \%$ e sem a sua utilização de $11 \pm 2 \%$, não sendo os valores médios estatisticamente diferentes.

Comparando a [em] entre diferentes formas de locomoção no meio aquático, Pendergast et al. (32) verificaram que esta era de $17 \%$ na Canoagem e $19 \%$ no Remo, para uma [Pmet] de $0.5 \mathrm{~kW}$. Estes valores são substancialmente mais elevados do que aqueles obser vados em situação de nado completo, cingindo se aos $8 \%$ e, no caso do nado exclusivamente com a acção dos membros inferiores, aos $4 \%$.

\subsection{0 trabalho mecânico e a potência relacionada com o arrasto}

Para haver deslocamento de um corpo biológico é necessário que ele realize trabalho mecânico externo. Acresce que no meio aquático também é necessário vencer a força de arrasto hidrodinâmico. Logo, neste meio, o trabalho necessário para vencer o arrasto é igual ao produto da intensidade desta força pelo deslocamento (29):

$\mathrm{W}=\mathrm{D} \cdot \mathrm{d}$

Onde [W] é o trabalho mecânico, [D] a força de arrasto hidrodinâmico e [d] o deslocamento.

O arrasto hidrodinâmico é condicionado por diversos factores, os quais são apresentados na expressão newtoniana:

$\mathrm{D}=1 / 2 \cdot \rho \cdot A \cdot \mathrm{C}_{\mathrm{D}} \cdot \mathrm{V}^{2}$

Onde [D] é a força de arrasto hidrodinâmico, [ $\rho]$ a densidade do fluido, [v] a velocidade de deslocamento do corpo, [A] a área de secção transversa máxima do corpo na direcção do deslocamento e $\left[C_{D}\right]$ o coeficiente de arrasto (grandeza que exprime a dependência da força relativamente às variáveis independentes $[\rho]$, $[v]$ e $[\mathrm{A}])$.

Então, segundo Nigg (29) o trabalho mecânico efectuado por [D] é:

$W=D \cdot d=1 / 2 \cdot \rho \cdot A \cdot C_{D} \cdot v^{2} \cdot d$

Ao analisar a taxa de trabalho mecânico realizado por unidade de tempo, ou seja, a potência necessária para vencer a força de arrasto hidrodinâmico (Pd):

$P d=$ D.v $=1 / 2 \cdot \rho \cdot A \cdot C_{D} \cdot v^{2} \cdot v=1 / 2 \cdot \rho \cdot A \cdot C_{D} \cdot v^{3}$

Portanto, a potência requerida para vencer a força de arrasto hidrodinâmico depende fundamentalmente do cubo da velocidade de deslocamento.

A força de arrasto e, portanto, o trabalho mecânico realizado, podem ser avaliados por meio de arrasto passivo (o nadador é rebocado na posição horizontal) ou por meio de arrasto activo (o sujeito deslocase autonomamente no meio aquático através de acções propulsivas). Foram descritas diversas metodologias de avaliação do arrasto activo, como a de di Prampero et al (16), a de Hollander et al. (20) ou a de Kolmogorov e Duplishcheva (27). Com efeito, segundo alguns autores, o arrasto activo é superior ao arrasto passivo (34). A alteração da área de secção transversa do corpo do sujeito na direcção do deslocamento ao longo do ciclo gestual, bem como a variação do tipo de escoamento do fluido em torno do nadador, devido às acções dos segmentos propulsivos e do tronco, motivarão o aumento do arrasto activo. Contudo, este facto não parece ser consensual, na medida em que também foi obser vado que o arrasto passivo poderia apresentar valores próximos do arrasto activo $(20,27)$.

\subsection{A propulsão e a energia cinética adicionada à água}

Durante a locomoção no meio terrestre, durante a fase de apoio do pé, o solo não absor ve quantidades significativas de energia cinética. Pelo menos em comparação com alguns casos particulares, como seja, a título ilustrativo, a locomoção em areia mole (49). No meio terrestre, grande parte se não toda a propulsão gerada pelo indivíduo, é utilizada para o 
seu deslocamento. Já no meio aquático uma parte da propulsão gerada é utilizada para vencer a força de arrasto hidrodinâmico (2). Outra parte é dissipada pela transferência de energia cinética para a camada de água envolvente do nadador $(\mathrm{Pk})$, acelerando a massa de água. Ou seja, para haver deslocamento no meio aquático é necessário deslocar a massa de água sensivelmente para trás. Assim, à massa de água "empurrada" é alterada a velocidade, criando um impulso (42). Assim a propulsão gerada é registada a partir do impulso produzido na massa de água:

$I=\int_{0}^{t} P d t=\frac{1}{t} \cdot \sum_{i=1}^{n}$ mi.vi

Em que [I] é o impulso produzido na massa de água, [P] é a propulsão, [mi] a massa de água deslocada, [vi] a velocidade adicionada à água e [t] o tempo. Entretanto, a propulsão é uma força especialmente determinada por factores hidrodinâmicos, como a velocidade segmentar, a velocidade de escoamento da água sobre os segmentos propulsivos, a orientação espacial dos deslocamentos segmentares propulsivos, a posição dos dedos e os ângulos de orientação e de ataque dos segmentos propulsivos $(43,44)$. Isto faz com que a variação da energia cinética da água e a eficiência sejam consequência de relações muitos finas, dificilmente discrimináveis qualitativamente.

Como foi referido anteriormente, para haver propulsão é aumentada a velocidade da massa de água "empurrada". Esta maior velocidade tem como consequência a transferência de energia cinética do nadador para o meio líquido $(14,36,37)$ :

$\Delta \operatorname{Ecin}=\frac{1}{2} \cdot \sum_{i=1}^{\mathrm{n}} \operatorname{mi} \cdot(\Delta v i)^{2}$

Onde $[\Delta$ Ecin] é a energia cinética, [mi] a massa de água e [vi] a velocidade adicionada à massa de água. Se em cada ciclo gestual, parte da massa de água é acelerada, então (14):

$\operatorname{Pcin}=F G \cdot \frac{1}{2} \cdot \sum_{i=1}^{\mathrm{n}} \operatorname{mi} \cdot(\Delta v i)^{2}$
Onde [Pcin] é a potência gerada para produzir energia cinética, [FG] é a frequência gestual, [mi] a massa de água e [vi] a velocidade adicionada à massa de água.

\subsection{A potência mecânica}

$\mathrm{Na}$ maioria dos actos de locomoção aquática, como por exemplo o Remo ou a Canoagem, o trabalho mecânico interno é negligenciável (32). Contudo, no caso do nado sem barbatanas o trabalho mecânico interno já apresenta um papel importante (32). Isto porque no nado sem materiais auxiliares de propulsão o trabalho mecânico interno é determinante para a colocação relativa dos segmentos corporais e, portanto, contribui para a adopção de uma posição corporal mais favorável para a produção de propulsão e a diminuição do [D]. A potência mecânica total (Ptot) pode assumir -se como sendo a potência mecânica externa (Po). No entanto, Zamparo et al. (50, 51) verificaram que a utilização de barbatanas reduzia em $75 \%$ a potência interna, em comparação com a sua não utilização, valor que não será negligenciável. Considera-se como potência mecânica interna (Pint) a necessária para acelerar um dado segmento tendo como referência o centro de massa do corpo (19). Contudo, no caso do estudo da NPD, será mais adequado, sempre que possível, o estudo da potência mecânica total (32):

Ptot $=$ Pint + Pext

Assumindo uma velocidade constante, ou tão constante quanto possível do centro de massa, a [Pext] produzida pelo nadador corresponde à potência para vencer a força de arrasto nas suas diversas componentes (Pd) e a potência gerada para produzir energia cinética $(14,36)$ :

Pext $=$ Pd + Pcin

\subsection{A eficiência propulsiva}

Se nem toda a potência é utilizada para vencer a força de arrasto hidrodinâmico, é possível determinar a percentagem utilizada com esse fim. À taxa de [Pext] que é utilizada para vencer a força de arrasto chama-se de eficiência propulsiva $(2,14,24,36,46)$ : 
$\mathrm{ep}=\frac{\mathrm{Pd}}{\mathrm{Pext}} .100=\frac{\mathrm{Pd}}{\mathrm{Pd}+\mathrm{Pcin}} .100$

Uma das metodologias utilizadas para determinar a [Pext] e as suas componentes é o Measuring Active Drag (MAD) system (20). Este sistema permite que o nadador se desloque na água tendo como pontos de propulsão uma sucessão de apoios fixos. Estes apoios encontram-se a uma distância constante e ajustável entre si e a uma profundidade pré-definida, ao longo de $23 \mathrm{~m}$. Actualmente o mesmo grupo de investigação encontra-se a desenvolver uma nova versão do $M A D$ system que, entre outras alterações, é fixo à parede lateral em vez de ao fundo da piscina e com apoios de uma diferente geometria. Uma das limitações desta metodologia é a impraticabilidade de avaliar o efeito da acção dos membros inferiores na propulsão. Os apoios existentes destinam-se exclusivamente para as superfícies palmares, estando os membros inferiores unidos e suportados por uma pull-buoy. Outra limitação consiste na impossibilidade de avaliar outra técnica de nado que não a técnica de crol.

Com o MAD system, assumindo uma velocidade constante, a força propulsiva média é equivalente à força de arrasto. Assim, praticamente nenhuma percentagem da [Pext] será perdida por transferência da mesma para a água $($ Ecin $=0$ ), pelo que o consumo de oxigénio medido ao nadar no MAD system reflecte a potência necessária para vencer a força de arrasto (37). Já a medição do consumo de oxigénio em nado livre indica a potência para vencer a força de arrasto e a potência necessária para transferir energia cinética para a água. Transferência essa, à massa de água envolvente ao nadador, que ocorre devido às acções segmentares no sentido de promover o deslocamento. Consequentemente, a velocidades inferiores ao limiar anaeróbio, é possível determinar [Pcin] através da expressão (37):

$$
\text { Pcin }=\left(\mathrm{PVO}_{2 \text { livre }}-\mathrm{PVO}_{2 \mathrm{MAD}}\right) \cdot \mathrm{em}
$$

Onde [Pcin] é a potência necessária para transferir energia cinética para a água, $\left[\mathrm{PVO}_{2 \text { livre }}\right]$ é o equivalente de consumo de oxigénio em nado livre, $\left[\mathrm{PVO}_{2 \mathrm{MAD}}\right]$ é o equivalente de consumo de oxigénio no MAD system e [em] a eficiência mecânica Neste caso a [em] é estimada através do declive da recta de regressão entre a $[\mathrm{Pd}]$ e o $\left[\mathrm{VO}_{2 \mathrm{MAD}}\right]$. Por seu lado a $\left[\mathrm{PVO}_{2}\right]$ é determinada através da expressão (37):

$\mathrm{PVO}_{2}=1 / 60 . \mathrm{VO}_{2} \cdot 10^{3}[4.2(4.07 . \mathrm{R})]$

Onde $\left[\mathrm{PVO}_{2}\right]$ é o equivalente de consumo de oxigénio, $\left[\mathrm{VO}_{2}\right]$ é o consumo de oxigénio e $[\mathrm{R}]$ o quociente respiratório.

Uma outra limitação do MAD system decorre da impossibilidade de avaliar as variáveis em estudo a velocidades próximas das verificadas em competição. A necessidade de nadar abaixo do limiar anaeróbio, implica a adopção de velocidades inferiores às observadas em conte xto competitivo.

Para além da abordagem fisiológica utilizando o $M A D$ system, outras metodologias foram entretanto desenvolvidas. de Groot e van Ingen Schenau (14) utilizaram uma abordagem teórica, com base na estimação da força propulsiva gerada pela mão. Berger (5) utilizou um sistema tridimensional de análise de imagens. Contudo, os valores de [ep] determinados pela abordagem fisiológica foram superiores aos obtidos pela análise cinemática e não se verificou uma correlação significativa entre os pares de valores nos nadadores em estudo (5).

Toussaint et al. (37) analisaram um nadador olímpico através da abordagem fisiológica. Verificaram que na técnica de crol, a uma velocidade de $1.2 \mathrm{~m} . \mathrm{s}^{-1}$, a [ep] foi de 58\%. Mais interessante ainda, é que este resultado confirma os valores teóricos referidos por de Groot e van Ingen Schenau (14). Por seu lado, a [em] foi de $8.5 \%$, inferior aos $15 \%$ obser vados por Toussaint et al. (35) ao avaliarem nadadoras a realizar o ciclo de braçada num ergómetro que simula a acção dos membros superiores (arm cranking). Acresce que os autores verificaram uma correlação significativa de $\mathrm{r}=-0.84$ entre a velocidade de nado e a [ep]. Um maior predomínio de trajectos motores rectilíneos implicam uma menor [ep] do que trajectos do tipo latero -mediais. Por outras palavras, o predomínio da propulsão com origem na força de arrasto propulsiva parece produzir uma menor [ep] do que com origem na força ascensional propulsiva. Com efeito, o desperdício na transferência de energia 
cinética para a água é 5 a 6 vezes superior ao adoptarem-se trajectos motores do tipo rectilíneo (14). Assim, a técnica mais eficiente caracteriza-se por conduzir a quocientes entre a força ascensional propulsiva e a força de arrasto propulsivo superiores à unidade (3).

A [ep] parece ser um factor discriminativo do nível de desempenho dos sujeitos. Toussaint (38) comparou nadadores de elite com triatletas. Verificou que não havia diferenças significativas na [Pext] ou na [em]. Assumindo uma mesma [Pmet], a percentagem de energia transferida por questões térmicas não apresentava diferenças significativas entre os dois grupos. Os factores discriminativos entre os nadadores e os triatletas foram a [Pd] e a [Pcin]. A [Pd] foi significativamente superior nos nadadores de elite. Já a [Pcin] foi significativamente superior nos triatletas. Consequentemente, a [ep] foi significativamente superior nos nadadores de elite do que nos triatletas.

Toussaint et al. (37) teceram algumas considerações sobre a hipotética [ep] das diferentes técnica de nado. Os autores obser varam que a [ep] foi ligeiramente superior a $50 \%$ na técnica de crol. Para as restantes técnicas de nado os autores especularam que este valor seria inferior aos 50\%. Para tal, partiram do pressuposto que os nadadores estariam sujeitos a um maior [D] e à ocorrência de uma maior [Pcin] a costas, a bruços e a mariposa do que a crol. Por outras palavras, a técnica de crol é aquela que apresentará uma maior [ep]. Todavia o estudo dos valores concretos da [ep] das restantes técnicas de nado, até ao momento, não foi objecto de exploração. A utilização de equipamentos como barbatanas pequenas e flexíveis permite um aumento da [ep] de $61 \%$ para $70 \%(50,51)$. A explicação reside numa diminuição da [Pint] em aproximadamente $75 \%$ e da [Pcin] em 40\% (51). Estas diminuições serão motivadas pela diminuição da frequência de pernada, o que induz uma menor velocidade de contracção muscular e uma maior eficiência na produção de força. No mesmo sentido, a utilização de palas faz com que, para uma mesma velocidade média de deslocamento, a [ep] aumente sensivelmente $7.8 \%$, motivado pela diminuição em $6 \%$ da [Pmet] e em 7.6\% da [Pext] (40). Quer utilizando palas, quer barbatanas, para uma mesma [Pext], ocorre um aumento da velocidade de deslocamento.
Consequentemente, o nado com palas e/ou barbatanas pode ser um meio especifico de treino da força de nadadores. No meio terrestre existem equipamentos de locomoção, como a bicicleta ou os patins, que permitem um aumento da economia e da velocidade de deslocamento a uma dada [Pmet] (28). No meio aquático, as barbatanas pequenas e flexíveis, assim como as palas, parecem ter o mesmo efeito. A [ep] nas actividades náuticas (Canoagem e Remo) é superior à verificada nas actividades aquáticas (nado completo, nado por acção exclusiva da pernada ou nado com barbatanas). Pendergast et al. (32), para uma mesma [Pmet] de $0.5 \mathrm{~kW}$, obser varam uma [ep] de $70 \%$ para as duas actividades náuticas estudadas, $58 \%$ para o nado com barbatanas, $45 \%$ para o nado completo e $36 \%$ para o nado por acção exclusiva dos membros inferiores.

Comparando seres humanos com animais aquáticos, existem diferenças substanciais na [ep]. Na técnica de nado aparentemente mais eficiente, o ser humano apresenta uma [ep] ligeiramente superior a 50\%. Por seu lado, a [ep] de peixes, como por exemplo de atuns, a deslocarem-se a um comprimento corporal por segundo será superior a esse valor (15). O motivo poderá ser a maior superfície propulsiva dos animais aquáticos, aliado ao menor coeficiente de arrasto de todo o corpo, em comparação com a dos seres humanos.

Uma das limitações da determinação da [Pcin], através do MAD System, é a incapacidade de conhecer o contributo da acção dos membros inferiores para a sua produção. Zamparo et al. (51) procuraram estimar o valor da [Pcin], nas técnicas ondulatórias, a partir do número de Froude (indicador da eficiência do movimento ondulatório):

$$
\mathrm{nF}=\frac{\mathrm{Pd}}{\mathrm{Pd}+\mathrm{Pcin}}
$$

Onde [nF] é o número de Froude, [Pd] a potência para vencer o arrasto e [Pcin] a potência necessária para transferir energia cinética para a água.

e

$\mathrm{nF}=\frac{\mu+\mathrm{v}}{2 \mu}$ 
Onde [nF] é o número de Froude, $[\mu]$ a velocidade de oscilação e [v] a velocidade de deslocamento. Logo,

$$
\frac{P d}{P d+P \operatorname{cin}}=\frac{\mu+v}{2 \mu}
$$

Assim, conhecendo a [Pd], a velocidade de deslocamento do corpo e a velocidade de oscilação, é possível determinar a [Pcin].

A [Pext] também pode ser calculada por meio da análise cinemática de imagens $(8,9)$. Para tal há que aceitar dois pressupostos: (i) que a [Pd] é equivalente ao produto da força propulsiva efectiva aplicada na mão pela velocidade de nado e; (ii) que a [Pcin] consiste no somatório do produto da força propulsiva efectiva pela velocidade do corpo do nadador, com a diferença entre a força resultante e a força propulsiva efectiva, ao que este somatório é multiplicado pela velocidade da mão.

Então, é possível determinar [em] pela expressão $(8,9)$ :

$\mathrm{em}=\frac{\mathrm{Re} \cdot \mathrm{V}_{\text {corpo }}+(\mathrm{R}-\mathrm{Re}) \cdot \mathrm{Vmão}}{\mathrm{Pi}}$

Onde [Pmet] é a potência metabólica, [R] é a força propulsiva total, [Re] é a força propulsiva efectiva aplicada na mão, $\left[\mathrm{v}_{\text {corpo }}\right.$ ] a velocidade média de deslocamento do centro de massa e $\left[\mathrm{v}_{\text {mão }}\right.$ a velocidade média de deslocamento da mão.

Logo, a expressão que permite calcular a $[\mathrm{ep}]$ é $(8,9)$ :

$$
\mathrm{ep}=\frac{\mathrm{Re} \cdot \mathrm{V}_{\text {corpo }}}{\operatorname{Re} \cdot \mathrm{V}_{\mathrm{corpo}}+(\mathrm{R}-\mathrm{Re}) \cdot \mathrm{Vmão}^{\mathrm{o}}}
$$

Ao estudar nadadores especialistas em diferentes distâncias, Cappaert et al. (8), verificaram que nos nadadores que nadavam ao ritmo específico da prova onde eram especialistas a [ep] apresentava valores significativamente diferentes. Os especialistas em provas mais longas apresentavam valores médios de [ep] significativamente superiores $(61.5 \pm 10.2 \%)$ do que os especialistas em provas de médias distância $(55.9 \pm 10.1 \%) \quad$ ou de que os especialistas em provas mais curtas $(47.8 \pm 7.1 \%)$. Todavia, não obser varam diferenças significativas na [em] entre os três grupos de nadadores.

\subsection{A eficiência total}

Toussaint et al. (37) apresentaram um sistema de avaliação da eficiência com base no [Pext]. Contudo, os mais recentes desenvolvimentos nesta área foram produzidos por Zamparo et al. (51). Estes autores desenvolveram um modelo de avaliação mais completo, pelo menos para a NPD, baseado no cálculo das três componentes da [Ptot].

De acordo com Pendergast et al. (32) se na maioria das actividades associadas à locomoção aquática a [Pint] é negligenciável, no caso da NPD ela parece apresentar um peso considerável; assim como no nado com barbatanas (51). Consequentemente, o estudo da potência produzida pelo nadador tomando em consideração exclusivamente a [Pext], subestimará os valores de [Ptot].

A taxa de potência metabólica disponibilizada para a potência mecânica total, define a eficiência total (eo):

et $=\frac{\text { Ptot }}{\text { Pmet }} .100$

Zamparo et al. (51) ao compararem o deslocamento, com e sem barbatanas, verificaram que a [et] era de $13 \pm 2 \%$ na primeira situação e de $11 \pm 2 \%$ na segunda situação. Já Pendergast et al. (32) apresentaram um valor de aproximadamente $18 \%$ para o crol completo e de $10 \%$ para a pernada de crol. Logo, a utilização de equipamentos como barbatanas tende a aumentar a eficiência total de nado. Será interessante futuramente determinar se a utilização de palas terá o mesmo efeito, no sentido de aumentar a [et] de nado. Com base no comportamento da [ep] utilizando palas (40) será admissível especular que a [et] tenderá a aumentar com a utilização dos ditos materiais auxiliares.

A [et] na locomoção humana no meio aquático por meio do nado foi inferior à verificada utilizando embarcações para o deslocamento no mesmo meio. A [et] variou entre os $10 \%$ no nado com barbatanas e os $27 \%$ no Remo, assumindo uma mesma [Pmet] para todas as actividades estudadas (32). 


\section{CONCLUSÕES}

Em resumo, no caso da locomoção aquática por meio do nado: (i) a [em] é bastante reduzida devido ao incremento do gasto energético para a termoregulação; (ii) a [em] não apresenta diferenças significativas de acordo com o nível de desempenho ou o sexo; (iii) a [ep] e a [et], na NPD, quando comparadas com outros meios de locomoção aquática são bastante reduzidas, devido a uma maior transferência de energia cinética para a água; (iv) a [ep] é um factor discriminativo do nível de desempenho dos sujeitos e; $(v)$ a [ep] evidencia aumentos com a utilização de barbatanas e de palas.

\section{CORRESPONDÊNCIA}

Tiago Barbosa

Instituto Politécnico de Bragança

Departamento de Ciências do Desporto

e Educação Física

Campus de Sta. Apolónia

Apartado 1101

5301-856 Bragança

Portugal.

barbosa@ipb.pt 


\section{REFERÊNCIAS}

1. Abrantes J (1986). Biomecânica do comportamento humano: formulação e validade de um modelo numérico aplicado ao padrão motor impulsão dinâmica unilateral. Dissertação de Doutoramento. Lisboa: Faculdade de Motricidade Humana da Universidade Técnica de Lisboa

2. Alexandre $M$ (1977). Swimming. In: Alexander M, Goldspink G (eds.). Mechanics and energetics of animal locomotion. New York: Wiley \& Sons, 222-254

3. Alves F (1995). Economia de nado e prestação competitiva - determinantes mecânicas e metabólicas nas técnicas alternadas. Dissertação de Doutoramento. Lisboa: Faculdade de Motricidade Humana da Universidade Técnica de Lisboa

4. Alves, F (1996). Economia de nado, técnica e desempenho competitivo nas técnicas alternadas. Natação V(28): separata

5. Berger $M$ (1996). Force generation and efficiency in front crawl swimming. Dissertação de doutoramento.

Amsterdam: Faculty of Human Movement Sciences, Vrije Universiteit

6. Billat V (1998). Physiologie et méthodologie de l'entrainement. Paris: De Boeck Université Amsterdam

7. Caldwell G, van Emmerik C, Hamill J (2000). Movement proficiency: incorporating task demands and constrains in assessing human movement. In: Sparrow A (ed) Energetics of human activity. Champaign, Illinois: Human Kinetics, 6695.

8. Cappaert J, Bone J, Troup J (1992a). Intensity and performance related differences in propelling and mechanical efficiencies. In: Maclaren D, Reilly T, Lees A (eds.) Biomechanics and Medicine in Swimming VI. London: E \& FN Spon, 49-52

9. Cappaert J, Franciosi P, Langhand G, Troup J (1992b). Indirect calculation of mechanical and propelling efficiency during freestyle swimming. In: Maclaren D, Reilly T, Lees A (eds.) Biomechanics and Medicine in Swimming VI. London E \& FN Spon, 53-56

10. Cavanagh P, Kram R (1985). The efficiency of human movement - a statement of the problem. Med and Sci in Sports and Exerc 17(3): 304-308

11. Charbonnier J, Lacour J, Riffat J, Flandrois R (1975). Experimental study of the performance of competition swimmer . Eur J Appl Physiol 34 : 157-167

12. Craig A (1984). The basics of swimming. Swim Tech, FebApr: 22-27

13. Counsilman J (1967). The science of swimming. Englewood cliffs, New York: Prentice Hall

14. de Groot G, van Ingen Schenau G (1988). Fundamental mechanics applied to swimming: technique and propelling efficiency. In: Ungerechts B, Wilke K, Reischle K (eds.). Swimming Science V. Champaign, Illinois: Human Kinetics Books, 17-29

15. Dewar H, Graham J (1994). Studies of tropical tuna swimming performance in a large water tunnel. J Exp Biol 192: 13-31

16. di Prampero P, Pendergast D, Wilson D, Rennie, D (1974) Energetics of swimming in man. J Appl Physiol 37(1): 1-5

17. di Prampero P (1986). The energy cost of human locomotion on land and in water. Int J Sports Med 7: 55-72

18. di Prampero P (1988). Les limites théoriques de la performance. In: Rieu M, Barrault D (eds.). VIIIéme Seminaire de bioenergetique - Les limites de la performance humaine. Paris : INESP, 57-59

19. Fenn W (1930). Frictional and kinetic factors in the work of sprint running. Am J Physiol 92:583-611

20. Hollander A, de Groot G, van Ingen Schenau G, Toussaint $\mathrm{H}$, de Best H, Peeters W, Meulemans A, Schreurs A (1986). Measurement of active drag during crawl arm stroke swimming. J Sports Sci 4(1) : 21-30

21. Holmér I. (1974). Physiology of swimming man. Acta Phys Scand (407): Suppl.

22. Holmér I. (1983). Energetics and mechanical work in Swimming. In: Hollander A, Huijing P, de Groot G (eds.) Biomechanics and Medicine in Swimming. Champaign, Illinois: Human Kinetics Publishers, 154-164

23. Hudson J (1991). Recidivists biomechanics; reading the questions. In: Wilkessn J, Kreighaum E, Tant C (eds.) Teaching Kinesiology and Biomechanics in sports. Ames: Iowa State University , 3-6

24. Huijing P, Hollander A, de Groot G. (1983). Efficiency and specificity of training in swimming: an editorial. In: Hollander A, Huijing P, de Groot G (eds.) Biomechanics and Medicine in Swimming. Champaign, Illinois: Human Kinetics Publishers, 1-6

25. Karpovich P (1933). Water resistance in swimming. Res Quart 4: 21-28.

26. Kemper H, Verschuur R, Clarys JP, Jisk oot J, Rijken, H (1976). Efficiency in swimming the front crawl. In: Komi PV (ed.). Biomechanics $V$-B. Baltimore: University Park Press, 243-249

27. Kolmogorov S, Duplishcheva O (1992) Active drag, useful mechanical power output and hydrodynamique force coefficient in different swimming strokes at maximal velocity. $J$ Biomech 25(3): 311-318

28. Minetti A, Pinkerton J, Zamparo P (2001). From bipedalism to bicyclim: evolution in energetics and biomechanics of historical bicycles. Proc R Soc Lond B268: 1351-1360.

29. Nigg B (1983). Selected methodology in biomechanics with respect to swimming. In: Hollander A, Huijing $P$, de Groot G (eds.) Biomechanics and Medicine in Swimming. Champaign, Illinois: Human Kinetics Publishers, 72-80

30. Pendergast D, di Prampero P, Craig A, Wilson D, Rennie D (1977). Quantitative analysis of the front crawl in men and women. J Appl Physiol 43(3): 475-479.

31. Pendergast D, di Prampero P, Craig A, Rennie D (1978). The influence of some selected biomechanical factors on the energy cost of swimming. In: Eriksson B, Furberg B (eds.) Swimming Medicine IV. Baltimore, Maryland: University Park Press, 367-378

32. Pendergast D, Zamparo P, di Prampero D, Capelli C, Cerrettelli P, Termin A, Craig A, Bushnell D, Paschke D, Mellendorf J (2003). Energy balance of human locomotion in water. Eur J Appl Physiol 90: 377-386.

33. Rodríguez F (1999). Cardiorespirator y and metabolic field testing in swimming and water polo: from physiological concepts to practical methods. In: Keskinen K, Komi P, Hollander A (eds.) Biomechanics and Medicine in Swimming VIII. Jyväsk ylä: Gummerus Printing, 219-226

34. Strojnik V, Bednarik J, Strumbelj B (1999). Active and passive drag in swimming. In: Keskinen K, Komi P, Hollander A (eds.) Biomechanics and Medicine in Swimming VIII. Jyväsk ylä: Gummerus Printing, 113-117

35. Toussaint H, van der Helm F, Elzerman J, Hollander A, de 
Groot G, van Ingen Schenau G (1983). A power balance applied to swimming. In: Hollander A, Huijing P, de Groot $\mathrm{G}$ (eds.) Biomechanics and Medicine in Swimming.

Champaign, Illinois: Human Kinetics Publishers, 165-172

36. Toussaint $H$ (1988). Mechanics and energetics of swim ming. Dissertação de Doutoramento. Faculty of Human Movement Sciences of the Vrije University Amsterdam. Amsterdam: Enschede

37. Toussaint $\mathrm{H}$, Hollander A, de Groot G, van Ingen Schenau G, Vervon K, de Best H, Meulemans T, Schreurs W (1988). Measurement of efficiency in swimming man. In: Ungerechts B, Wilke K, Reischle K (eds.) Swimming Science V. Champaign, Illinois: Human Kinetics Books, 45-52

38. Toussaint H (1990). Differences in propelling efficiency between competitive and triathlon swimmers. Med Sci Sports Exerc 22(3): 409-415

39. Toussaint H, Knops W, de Groot G, Hollander A (1990). The mechanical efficiency of front crawl swimming. Med Sci Sports Exerc 22(3): 402-408

40. Toussaint H, Janssent T, Kluft M (1991). Effect of propelling surface size on the mechanics and energetics of front crawl swimming. J Bioech 24(3-4): 205-211

41. Toussaint H (1992). Performance determining factors in front crawl swimming. In: Maclaren D, Reilly $\mathrm{T}$, Lees A (eds.) Biomechanics and Medicine in Swimming VI. London: E \& FN Spon, 13-32

42. Toussaint, H, Hollander A (1994). Mechanics and energetics of front crawl swimming. In: Miyashita M, Mutoh Y, Richardson A (eds.) Medicine and science in aquatic sports. Bassel: Karger, 107-116

43. Vilas-Boas JP (1987). O mecanismo propulsivo em Natação. Aula síntese apresentada nas Provas de Aptidão Pedagógica e Capacidade Científica. Porto: Faculdade de Ciências do Desporto e de Educação Física da Universidade do Porto

44. Vilas-Boas JP (1993). Caracterização biofísica de três variantes da técnica de Bruços. Dissertação de Doutoramento. Faculdade de Ciências do Desporto e de Educação Física da Universidade do Porto. Porto: Medisa

45. Vilas-Boas, JP (2004). Relatório pedagógico da disciplina de Biomecânica. Provas de agregação do $3^{\circ}$ grupo de disciplinas. Porto: Faculdade de Ciências do Desporto e de Educação Física

46. Webb P (1971). The swimming energetics of trout. Oxygen consumption and swimming efficiency.J Exp Biol 55: 521-540

47. Williams K (1985). The relationship between mechanical and physiological energy estimates. Med Sci Sports Exerc 17(3): $317-325$

48. Winter D (1990). Biomechanics and motor control of human movement. Chichester : John Wiley \& Sons

49. Zamparo P, Perini R, Orizio C, Sacher M, Ferretti G (1992). The energy cost of walking or running on sand. Eur J Appl Physiol Occup Physiol 65(2): 183-187

50. Zamparo P, Pendergast D, Termin B, Minetti E. (2001). Energy cost and efficiency swimming with fins. In: Mester J, King G, Struder H, Tsolakidis E, Osterburg A (eds.) Book of Abstracts of the $6^{\text {th }}$ Annual Congress of the European College of Sports Sciences. Cologne: Sport und Buch Strauss, 531

51. Zamparo P, Pendergast D, Termin B, Minetti E (2002). How fins affect the economy and efficiency of human swimming. J Exp Biol 205: 2665-2676. 